\title{
The larynx ruler to measure height and profile of vocal folds: a proof of concept
}

This article was published in the following Dove Press journal:

Medical Devices: Evidence and Research

5 July 2017

Number of times this article has been viewed

\author{
Gauthier Desuter, ${ }^{1,2}$ \\ Benjamin Mertens, ${ }^{3}$ \\ Alain Delchambre, ${ }^{3}$ \\ Julie van Lith-Bijl, ${ }^{1,4}$ \\ Peter Paul van Benthem, ${ }^{2}$ \\ Elisabeth Sjögren² \\ 'Otolaryngology, Head \& Neck \\ Surgery Department, Voice \& \\ Swallowing Clinic, Cliniques \\ universitaires Saint-Luc, Université \\ catholique de Louvain, Brussels, \\ Belgium; ${ }^{2}$ Otolaryngology, Head \& \\ Neck Surgery Department, LUMC, \\ University of Leiden, Leiden, the \\ Netherlands; 'BEAMS Department, \\ Ecole Polytechnique de Bruxelles, \\ Université libre de Bruxelles, \\ Brussels, Belgium; ${ }^{4}$ Otolaryngology \\ Department, Flevoziekenhuis, Almere, \\ the Netherlands
}

Introduction: Glottic leakage during phonation is a direct consequence of unilateral vocal fold (VF) paralysis. This air leakage can be in the horizontal plane and in the vertical plane. Presently, there is no easily applicable medical device allowing noninvasive, office-based measurement of the relative vertical position of the VFs. The larynx ruler (LR) is a laser-based measuring device that could meet the previously stated need, using a flexible endoscope. This study represents a proof of concept regarding the use of the LR in assessing VF relative positions in the vertical plane.

Materials and methods: One fresh male human cadaver larynx, free of neurologic and anatomic disease, was explored with the LR system through the operative channel of a flexible gastroenterology video-endoscope. The tip of the video-endoscope was located in the laryngeal vestibule. The right crico-arytenoid joint was posteriorly disarticulated. Tilting of the VF was obtained by pulling or pushing the arytenoid cartilage with a mosquito forceps fixed to the stump of the previously sectioned superior tip of the posterior crico-arytenoid muscle allowing anterior and posterior tilting of the arytenoid cartilage in order to induce an elevation or a depression of the VF process. Ten "push" and ten "pull" sessions were performed. The distance from the tip of the video-endoscope to each illuminated pixel of the laser beam was recorded. The level difference between the left and right VFs was measured for each recording.

Results: Data provided by the LR were consistently in accordance with the movements applied on the VFs. The accuracy of $0.2 \mathrm{~mm}$ of the LR is compatible with the envisioned applications for the human larynx.

Conclusion: The LR system represents a feasible technique to evaluate respective vertical position of VFs in the human larynx. Technical limitations were identified that will require improvements before experimental use on human beings.

Keywords: laryngoscopy, laser, measurement device, unilateral vocal fold paralysis

\section{Introduction}

During phonation, the closed vocal folds (VFs) offer resistance to the air expelled by the lungs during expiration. Their vibro-elastic properties allow for the interruption of the air flow that eventually will lead to vibration of the VFs and creation of sound.

The VFs are located in the midpart of the thyroid cartilage. Their common anterior insertion is fixed at the anterior commissure. Their posterior anchoring corresponds to the vocal process of each arytenoid cartilage and is mobile in the three dimensions. The position of the vocal process is determined by the balanced contraction of all intrinsic laryngeal muscles under the neurological control of the vagal nerve and its terminal branches. Under normal conditions, the position of both vocal processes will
Correspondence: Gauthier Desuter Otolaryngology, Head \& Neck Surgery Department, Voice \& Swallowing Clinic, Cliniques universitaires St-Luc, 10 avenue Hippocrate, suite 407, 1200 Brussels, Université catholique de Louvain, Brussels, Belgium

Tel +32 2764 I355

$\mathrm{Fax}+3237648935$

Email gauthier.desuter@uclouvain.be 
be located at equal distance from any reference point located at the midline of the larynx.

In the case of innervation unbalance of the larynx, for example in unilateral vocal fold paralysis (UVFP), the abovementioned symmetry will be disrupted and may result in incomplete glottic closure during phonation, leading to leakage of air at the level of the glottis. This innervation unbalance of the larynx will not only lead to a closure defect in the horizontal or axial plane but possibly also, in the vertical or coronal plane. This is why determination of the exact threedimensional (3D) position of the posterior part of the VF is key when it comes to preoperative planning of UVFP treatment.

As a matter of fact, the best treatment strategy to obtain optimal voice outcome after UVFP treatment is still debated. Most discussions concentrate on addressing posterior leakage. For instance, some authors advocate a systematic arytenoid adduction strategy, while others deny any use of it at all. ${ }^{1-6}$ The truth is that no evidence exists and the question remains unanswered. . $^{7}$

Determination of the exact $3 \mathrm{D}$ position of the VF before and after treatment could help to answer the above-mentioned question. Three-dimensional computed tomography (CT) imaging has shown its value in tackling this problem but requires patient irradiation and delayed image processing. Presently, there is no device allowing real-time, harm-free and office-based quantification of the vertical relative position of the VF. Industry is developing 3D video-endoscopic imaging but quantification of exact VF position remains impossible.

The larynx ruler (LR) is a laser-based endoscopically guided, measuring device that could meet the previously stated needs.

The primary purpose of this study is to see whether the LR technology could measure relative VF height (or level) differences, between the right and the left VF, measured from the tip of the endoscope.

\section{Materials and methods The laryngeal ruler device}

The LR device consists of a laser probe based on a miniaturized pattern projector placed in the instrument channel of the endoscope (Olympus GIF-100; Olympus Inc., Hamburg, Germany). It has a diameter of $2.7 \mathrm{~mm}$ (Figure 1) and projects a line used as reference for analysis.

\section{Principle of 3D reconstruction}

The LR is based on the structured light technology. A known pattern is projected and its image is analyzed to recover the $3 \mathrm{D}$ position of the points of interest (Figure 2). The projector

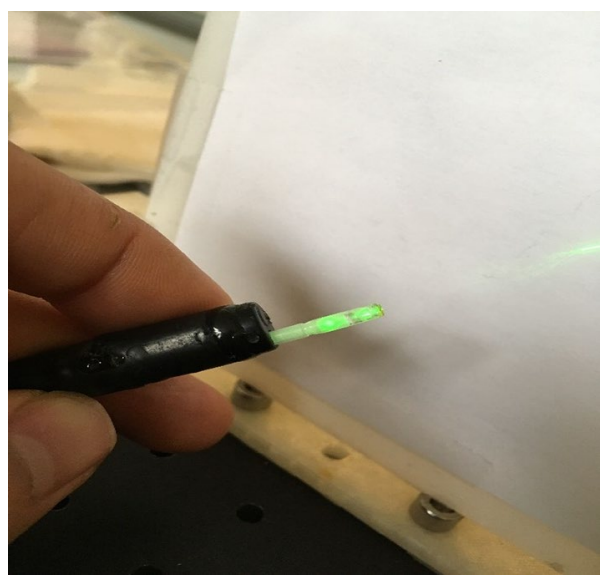

Figure I Laser probe placed in the instrument channel of the endoscope.

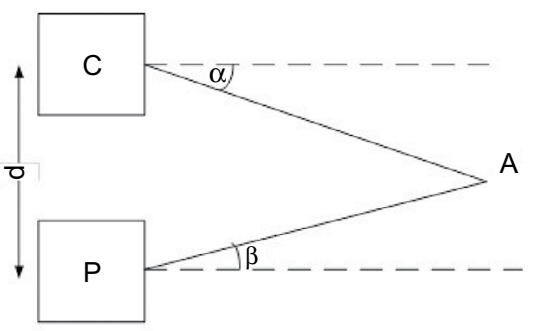

Figure 2 Point $A$ is found by triangulation knowing the baseline $d$ between camera (C) and the projector $(\mathrm{P})$ and the angles $\alpha$ and $\beta$.

$\mathrm{P}$ projects a laser line toward a point $\mathrm{A}$ on a surface and the camera $\mathrm{C}$ acquires the image. Depth ( $\mathrm{z}$ ) is then easily calculated as in the equation below, where $\alpha$ is the angle of projection measured using the pixel position of the point $\mathrm{A}$ as detailed, and $\beta$ is known by calibration. ${ }^{9}$ It is the angle of projection of the line in the camera coordinates, and the distance $d$ (between camera and projector) is the baseline known by calibration. ${ }^{10}$ It can be easily shown that:

$$
z=\frac{d}{\tan (\alpha)-\tan (\beta)}
$$

In this example, the system is brought back to a $2 \mathrm{D}$ system, but it can be generalized to a 3D system. More information about structured light $3 \mathrm{D}$ reconstruction can be found in the study by Mertens B. ${ }^{9}$

\section{Projection principle}

The projection is based on a diffracted laser beam using a diffractive optical element (DOE). DOEs are commercially available (eg, Holoeye, Berlin, Germany) and are engraved to project a pattern (in this case, a simple line) as a focused laser beam passes through it (Figure 3). The pattern used is a single line, placed perpendicularly to the epipolar plane. It enables a direct measurement of every point of the line.

Structured light was already used by several authors who have developed a device based on two laser dots that provide 


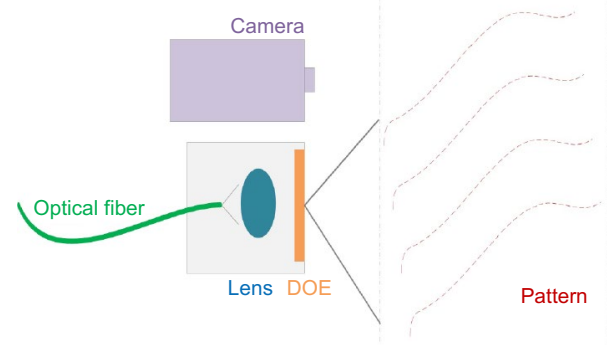

Figure 3 The system is composed of an optical fiber to transmit the beam, a lens to focus it, and a DOE to generate the pattern. The pattern is then analyzed using the camera of the endoscope.

Abbreviation: DOE, diffractive optical element.

a mean for estimating depth of the region of interest. ${ }^{11,12,13}$ However, in this solution, the measurements are based on approximations. If the region of interest is not flat, it could lead to relatively important errors. Neitsch et al have proposed a smart solution based on a matrix of points (instead of two points). ${ }^{14}$ The main limitation here is that measurements are possible only where points are projected (or detectable).

With regard to safety, the power density is lower than a conference laser pointer as the beam is used for a complete line, whereas a laser pointer is focused on a single point. The power density is of $16 \mathrm{~W} / \mathrm{m}^{2}$ with a total power lower than $1 \mathrm{~mW}$. The laser source has a maximum power of $20 \mathrm{~mW}$ at a wavelength of $532 \mathrm{~nm}$. LR is thus harm-free for the patient.

The accuracy of this technology has been demonstrated elsewhere. ${ }^{9}$

In a nutshell, the accuracy can be calculated by:

$$
\delta z=\frac{\delta p_{\mathrm{x}} z^{2}}{d f_{\mathrm{x}}}
$$

where

- $\delta z$ is the accuracy (in $m$ );

- $\delta \mathrm{p}_{\mathrm{x}}$ is the accuracy of the line detection (in pixel);

- $\mathrm{z}$ is the working depth (in $\mathrm{m}$ );

- $\mathrm{d}$ is the baseline (distance between the camera and the projector, in $\mathrm{m}$ );

- $\mathrm{f}_{\mathrm{x}}$ is the focal length of the camera (in pixel).

\section{Measuring process}

The probe is kept in place by friction; the diameter of the device being the same as the channel diameter. The user places the device in the instrument channel and calibrates it before performing the endoscopy. The user checks the calibration (a ruler is used as reference) before and after performing the endoscopy.
To proceed with the measurement, the following steps were followed:

1. The endoscopist places the laser line on the measurement site. The image is then frozen.

2. An external dedicated computer acquires the image and processes it to compute the data.

3. The user may pick up the points of interest for exact measurements.

4. Data are displayed instantaneously on the endoscopic image and the profile in a dedicated window.

A homemade dedicated software using the OpenCV library is used to compute the complete profile based on the equations mentioned above. The system is calibrated prior to any use to take the lens distortion and the tolerances of assembly into account. The processing time is below $50 \mathrm{~ms}$.

The generated profile is required to provide the user the ability to select exactly the relevant points of interests. In addition to that, the points of interests are shown on both the endoscopic image and the 3D profile to make sure the distances are relevant. Therefore, the user is able to select visually the points of interest by considering the anatomical structures on the endoscopic image itself.

However, the position of the tip of the endoscope (specially a possible angle-view) related to the larynx must be understood by the user to properly understand the generated profiles. The distance of the endoscope to the larynx has no influence as the profile is generated in 3D (it simply shifts the profile).

Height measurements at each pixel point of the laserilluminated structures open the field of 3D assessment of the larynx.

\section{Proof-of-concept measurements}

The study protocol was approved by the University of Louvain Institutional Ethical Committee under the number 2017/18JAN/028. The anatomical material was harvested according to Institutional Ethical Committee regulations.

One fresh unfrozen human male larynx was used for assessment of VF heights. According to patient medical file examination and anatomical inspection, the larynx was declared free of disease.

The specimen was examined with the LR system through the operative channel of a gastroenterology videoendoscope. The tip of the video-endoscope was positioned in the laryngeal vestibule. Experimental setting is detailed in Figure 4. 


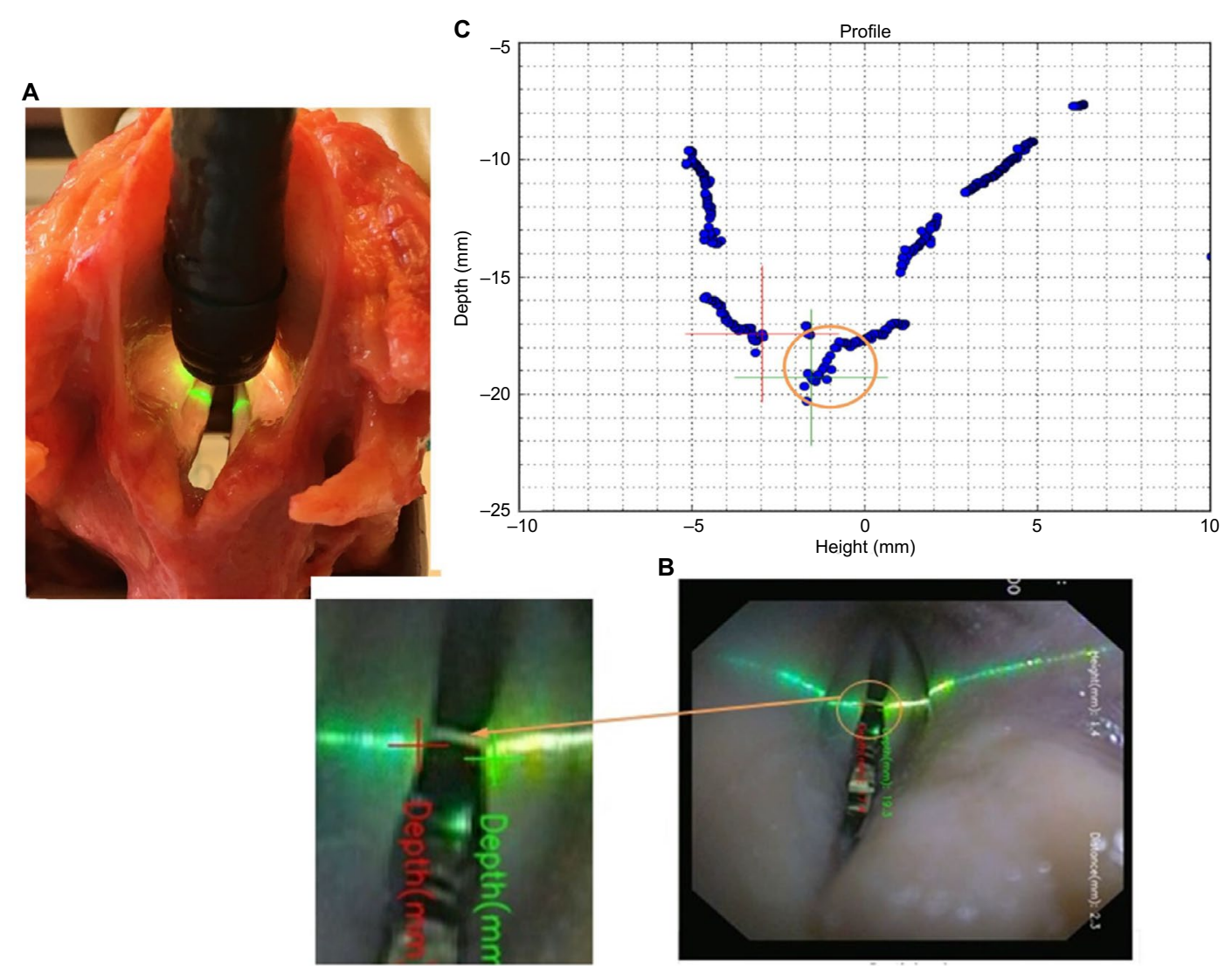

Figure 4 (A) Study setting showing the operative video-gastroscope introduced into the larynx vestibule with the laser beam "scanning" both vocal folds. (B) Endoscopic image of the laser beam "scanning" the glottis and the larynx vestibule; a zoomed image shows the respective positions of VF medial edges where VF height was measured from the tip of the video-endoscope (crosses represent medial free edges [green=right, red=left]; note the fortuitous presence of a secretion between the vocal folds at the level of the laser beam). (C) Larynx ruler screen capture showing the section profile by plotting heights measured from the tip of the video-endoscope, of every pixel that is illuminated along the laser beam. The orange circle in (C) corresponds to the orange circle in (B).

Abbreviation: VF, vocal fold

The right crico-arytenoid joint of the larynx was posteriorly disarticulated allowing anterior and posterior tilting of the arytenoid cartilage in order to create an elevation or a depression of the VF process. Tilting of the VF was obtained by pulling or pushing the arytenoid cartilage with a mosquito forceps fixed to the stump of the previously sectioned superior tip of the posterior crico-arytenoid muscle. The experimentally induced "push-and-pull" motions were compared at a given time with VF distances measured from the tip of the endoscope provided with the LR technology. Ten "push" and ten "pull" sessions were performed. The distance from the tip of the video-endoscope to each illuminated pixel of the laser beam was recorded and plotted on a diagram (Figure 5). The level difference between the left and right VFs was measured for each recording.

A VF profile, showing the surface of the laser-illuminated VF, was realized during each measurement. Profile characteristics that were scrutinized were: 1) ease of finding the medial edge pixel of VFs; 2) seamless surface profile of the VF; and 3) identification of Morgagni's ventricle gap.

\section{Results}

The results are shown in Table 1. There was a perfect correlation between the relative position of the VFs (above or below the contralateral control side) and the relative heights measured by the LR technology. In other words, using the LR, it was possible to detect and quantify the difference in VF heights, measured from the tip of the video-endoscope, induced by manipulating the arytenoid cartilage. The force applied on the arytenoid cartilage was different for each session; therefore, the value of the height difference was variable. Likewise, the position of the tip of the endoscope was not completely fixed; thus, the absolute value of the VF height was different for each measurement.

The profiles were measured during the endoscopy and were available for post-checking after the endoscopy.

A profile (or shape) of the surface of the VF at the level of the LR laser beam was always obtainable. This profile offers a visualization of a virtual coronal section of the VF at the level of the LR's laser beam (Figure 6). 

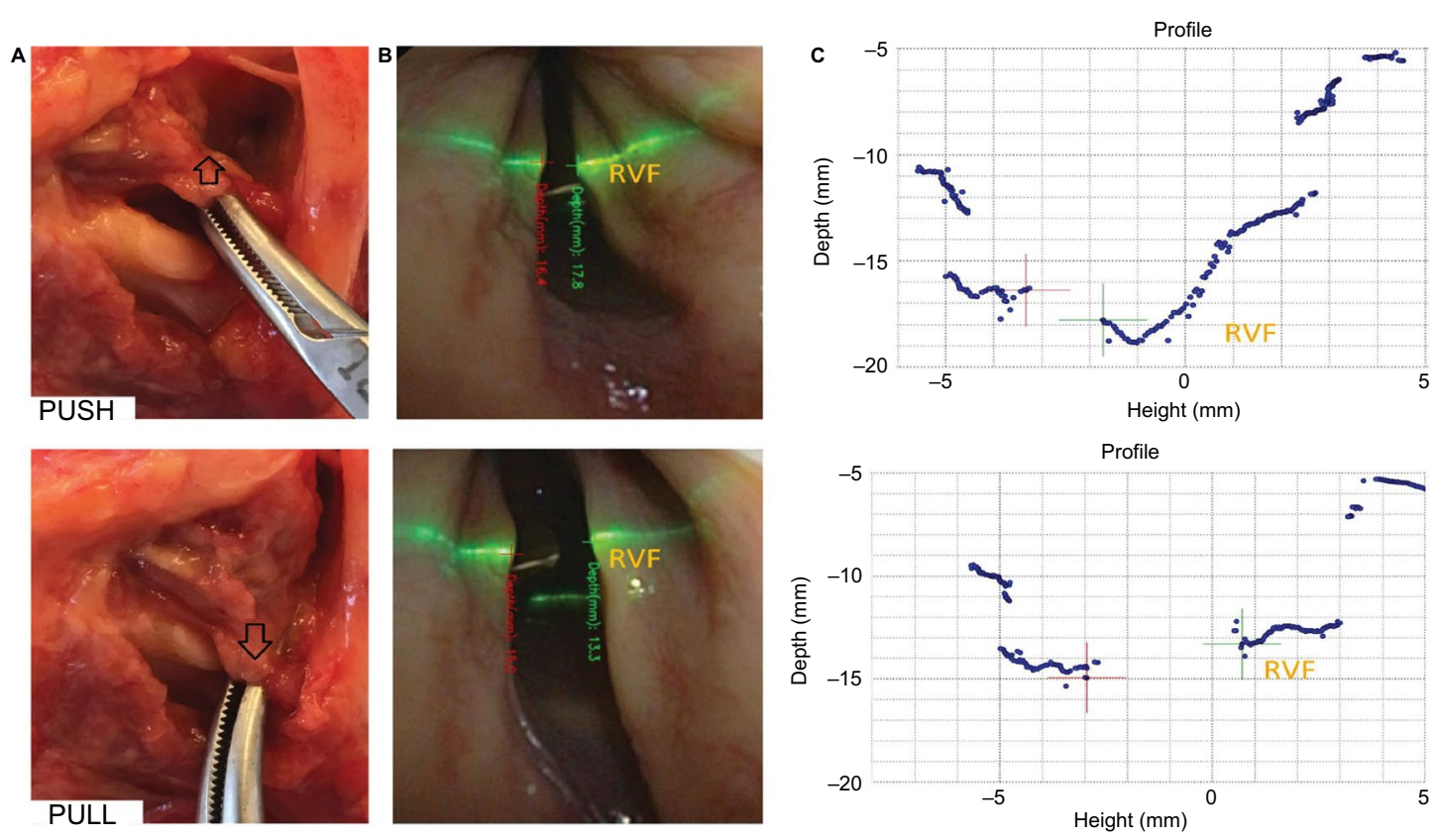

Figure 5 Simultaneous images of, (A) the arytenoid motion applied on the specimen by a mosquito forceps on the posterior crico-arytenoid muscle stump. The 'push motion' opens the crico-arytenoid joint and glides the arytenoid cartilage down internally on the cricoid cartilage. This lowers the VF. The 'pull motion' glides the arytenoid posteriorly on the cricoid crest. This elevates the VF. (B) The endoscopic image obtained with the LR video-endoscope showing the laser beam position. (C) The corresponding screen capture provided by the LR. Note the difference of heights between VFs visualized and quantified on the screen capture images. These differences are not clearly visible on two-dimensional endoscopic images.

Abbreviations: LR, larynx ruler; VF, vocal fold; RVF, right vocal fold.

Table I VF heights from the tip of the video-endoscope and the relative position of the mobilized VF for each session

\begin{tabular}{|c|c|c|c|c|c|c|}
\hline \multirow[t]{2}{*}{ Sessions } & \multicolumn{3}{|c|}{ PUSH of the right arytenoid } & \multicolumn{3}{|c|}{ PULL of the right arytenoid } \\
\hline & $\begin{array}{l}\text { Left VF } \\
\text { height }(\mathrm{mm})\end{array}$ & $\begin{array}{l}\text { Right VF } \\
\text { height }(\mathrm{mm})\end{array}$ & $\begin{array}{l}\text { Relative position of } \\
\text { mobilized (right) } \\
\text { VF (mm) }\end{array}$ & $\begin{array}{l}\text { Left VF } \\
\text { height }(\mathrm{mm})\end{array}$ & $\begin{array}{l}\text { Right VF } \\
\text { height }(\mathrm{mm})\end{array}$ & $\begin{array}{l}\text { Relative position of } \\
\text { mobilized (right) } \\
\text { VF }(\mathbf{m m})\end{array}$ \\
\hline I & 17.6 & 19.5 & $1.9 \mathrm{~mm}$ below & 14.8 & 12.7 & 2.1 $\mathrm{mm}$ above \\
\hline 2 & 14.5 & 16.2 & $1.7 \mathrm{~mm}$ below & 14.2 & 12.9 & $1.3 \mathrm{~mm}$ above \\
\hline 3 & 15.9 & 16.8 & $0.9 \mathrm{~mm}$ below & 15.9 & 12.8 & $3.1 \mathrm{~mm}$ above \\
\hline 4 & 18.6 & 21.7 & $3.1 \mathrm{~mm}$ below & 12.4 & 10.4 & $2.0 \mathrm{~mm}$ above \\
\hline 5 & 20.0 & 22.5 & $2.5 \mathrm{~mm}$ below & 19.1 & 16.5 & $2.6 \mathrm{~mm}$ above \\
\hline 6 & 18.4 & 20.2 & $1.8 \mathrm{~mm}$ below & 16.7 & 14.8 & $1.9 \mathrm{~mm}$ above \\
\hline 7 & 16.4 & 17.8 & $1.4 \mathrm{~mm}$ below & 15.0 & 13.3 & $1.7 \mathrm{~mm}$ above \\
\hline 8 & 19.6 & 21.4 & 1.8 mm below & 16.8 & 14.0 & $2.8 \mathrm{~mm}$ above \\
\hline 9 & II.3 & 16.5 & $5.2 \mathrm{~mm}$ below & 13.6 & 12.2 & $1.4 \mathrm{~mm}$ above \\
\hline 10 & 15.0 & 17.9 & $2.9 \mathrm{~mm}$ below & 15.0 & 13.2 & $1.8 \mathrm{~mm}$ above \\
\hline
\end{tabular}

Abbreviation: VF, vocal fold.

VF heights could be measured in all the evaluated positions. In four of the 20 screen captures, a seamless profile was not obtainable. These profiles were discontinuous and did not allow a clear profiling of glottic surface. Despite these caveats, Morgagni's ventricle depths were always visible and quantifiable. No tissue damage was observed.

\section{Discussion}

Our results show that use of the LR is technically feasible in a human larynx. A perfect correlation of VF height differences between left and right VFs and imposed VF motions was found. Qualitative analysis showed that a VF profile was obtainable in all of the push-pull sessions. The aim of this proof of concept was not to assess the accuracy of the LR but rather to assess its possible utility in the laryngology field in general and in UVFP management in particular.

Nevertheless, in the case of our experiment in the larynx, with a distance between the LR and the VF of $\sim 2 \mathrm{~cm}$, the accuracy is about $0.2 \mathrm{~mm}$, which is compatible with the foreseen clinical applications of LR. Indeed, none of our height differences were $<0.9 \mathrm{~mm}$. 


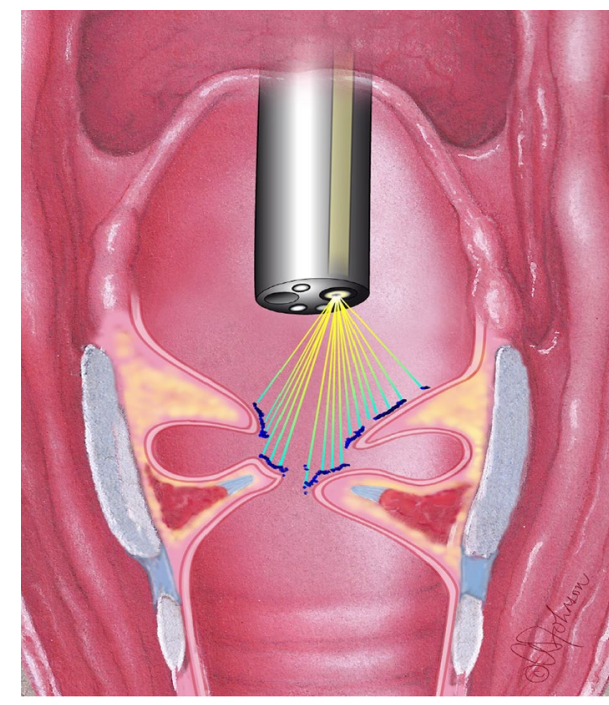

Figure 6 The relationship between the scanning laser beam and the "profile" offered by the larynx ruler data interpretations.

Note: Courtesy of Ann Johnson.

At present, there is considerable interest concerning the topic of 3D positioning of the VF where magnetic resonance imaging (MRI) and CT studies in patients have been performed as well as laryngoscopic high-speed camera imaging in animal models. ${ }^{15}$ MRI has a lower resolution, and CT scanner necessitates radiation exposure and post-acquisition software-based interpretation. ${ }^{16,17,18,19,20}$ Both these imaging techniques are costly and do not allow for the examination of patients under physiological conditions. The high-speed camera does not have the disadvantage of irradiation but requires two simultaneously introduced endoscopes and two high-speed cameras. Furthermore, it also requires postrecording analysis, so it does not yield real-time readings. Compared to prior articles using the same technology, apart from application using a flexible endoscope, this is the first experimentation with a continuous line and it enables a fine analysis of the VF with the generated profile. ${ }^{11,12,13,14}$

Profiling of the explored surfaces can be realized using multiplication of the acquired measures. As shown in Figure 6, profiling of VF surface could possibly reflect and quantify the degree of thyroarytenoid atrophy in cases of UVFP. Some of these profiles were discontinuous and tedious to read. Technical refinements and adaptations that are specific to the larynx anatomy should be done in order to offer clinically useful profiles.

Despite these encouraging results many challenges still have to be overcome before the LR is used in patients. The endoscope that was used is a gastroscope (11 $\mathrm{mm}$ of diameter), which is too large for intranasal use. A device with a maximum diameter of $1.9 \mathrm{~mm}$ should be developed to allow its use through the operative channel of Ear Nose and Throat flexible video-endoscope. Specific software for VF analysis and interpretation should be developed to improve the profile representations and height measurement in a more reliable way.

In our study models, the vocal processes were always easily visualized. However, in clinical UVFP situations, the vocal process on the paralyzed side can be difficult to visualize because of the anterior tilt of the arytenoid cartilage. Hypothetically, this hurdle could be overcome by the development of software that would, 1) measure various VF free edge points that can be effectively visualized, 2) draw a line through these points, 3) calculate the VF free edge slope, and eventually, 4) extrapolate the invisible VF depth based on the contralateral VF length.

\section{Conclusion}

Innovative technology is infrequent in the field of laryngology. This proof of concept could signify a new breakthrough. This study's results has allowed the authors to achieve their goal of demonstrating potential utility of the device. With the LR technology, a tool is acquired that allows a reliable, real-time, 3D position determination of the VFs, free of harm for the patient and instantaneously performed within an office setting. Therefore, it contributes to preoperative planning of laryngeal surgery and the postoperative follow-up. The LR technology deserves further clinical testing, even though several technical improvements are still required.

\section{Acknowledgments}

Authors are thankful to C Behets-Wydemans, B Caelen, and $\mathrm{M}$ van de Woestijne from the Department of Anatomy and Morphology of the University of Louvain and $F$ van Immerseel, J Aarents, and J den Boeft from the Department of Anatomy and Embryology at the Leiden University Medical Center for their assistance.

\section{Disclosure}

The authors report no conflicts of interest in this work.

\section{References}

1. Daniero JJ, Garrett CG, Francis DO. Framework Surgery for Treatment of Unilateral Vocal Fold Paralysis. Curr Otorhinolaryngol Rep. 2014; 2(2):119-130.

2. Hess MM, Fleischer S. Laryngeal framework surgery: current strategies. CurrOpin Otolaryngol Head Neck Surg. 2016;24(6):505-509.

3. Li AJ, Johns MM, Jackson-Menaldi C, et al. Glottic closure patterns: type I thyroplasty versus type I thyroplasty with arytenoid adduction. J Voice. 2011;25(3):259-264.

4. Mortensen M, Carroll L, Woo P. Arytenoid adduction with medialization laryngoplasty versus injection or medialization laryngoplasty: the role of the arytenoidopexy. Laryngoscope. 2009;119(4):827-831.

5. Woo P. Arytenoid adduction and medialization laryngoplasty. Otolaryngol Clin North Am. 2000;33(4):817-840. 
6. Benninger MS, Manzoor N, Ruda JM. Short- and long-term outcomes after silastic medicalization laryngoplasty: are arytenoid procedures needed? J Voice. 2015;29(2):236-240.

7. Siu J, Tam S, Fung K. A comparison of outcomes in interventions for unilateral vocal fold paralysis: a systematic review. Laryngoscope. 2016;126(7):1616-1624.

8. Chester MW, Stewart MG. Arytenoid adduction combined with medicalization thyroplasty: an evidence-based review. Otolaryngol Head Neck Surg. 2003;129(4):305-310.

9. Mertens B, Delchambre A. 3D reconstruction: why should the accuracy always be presented in the pixel unit? Image Vis Comput. 2016;48: 57-60.

10. Geng J. Structured-light 3d surface imaging: a tutorial. Adv Opt Photonics. 2011;3(2):128-160.

11. Herzon GD, Zealear DL. New laser ruler instrument for making measurements through an endoscope. Otolaryngol Head Neck Surg. 1997;116(6 Pt 1):689-692.

12. Kuo CF, Wang HW, Hsiao SW, et al. Development of laryngeal video stroboscope with laser marking module for dynamic glottis measurement. Comput Med Imaging Graph. 2014;38(1):34-41.

13. Schade G, Hess M, Rassow B. [Possibility for endolaryngeal morphometric measurements with a new laser light method]. HNO 2002;50(8):753-755. German
14. Neitsch M, Horn IS, Hofer M, Dietz A, Fischer M. Integrated multipointlaser endoscopic airway measurements by transoral approach. Biomed Res Int. 2016;2016:6838697.

15. Vahabzadeh-Hagh AM, Zhang Z, Chhetri DK. Quantitative evaluation of the in vivo vocal fold medial surface shape. J Voice. 2017. pii S0892-1997(16)30320-30324.

16. Vachha BA, Ginat DT, Mallur P, Cunnane M, Moonis G. "Finding a voice": imaging features after phonosurgical procedures for vocal fold paralysis. AJNR Am J Neuroradiol. 2016;37(9):1574-1580.

17. Burdumy M, Traser L, Burk F, et al. One-second MRI of a threedimensional vocal tract to measure dynamic articulator modifications. J Magn Reson Imaging. Epub 2016 Dec 9.

18. Vorik A, Unteregger F, Zwicky S, Schiwowa J, Potthast S, Storck C. Three-dimensional imaging of high-resolution computer tomography of singers' larynges-a pilot study. J Voice. 2017;31(1):115.e17-e21.

19. Storck C, Juergens P, Fischer C, et al. Biomechanics of the cricoarytenoid joint:three-dimensional imaging and vector analysis. $J$ Voice. 2011;25(4):406-410.

20. Hiramatsu H, Tokashiki R, Kitamura M, Motohashi R, Tsukahara K, Suzuki M. New approach to diagnose arytenoid dislocation and subluxation using three-dimensional computed tomography. Eur Arch Otorhinolaryngol. 2010;267(12):1893-1903.
Medical Devices: Evidence and Research

\section{Publish your work in this journal}

Medical Devices: Evidence and Research is an international, peerreviewed, open access journal that focuses on the evidence, technology, research, and expert opinion supporting the use and application of medical devices in the diagnosis, monitoring, treatment and management of clinical conditions and physiological processes. The identification of novel

\section{Dovepress}

devices and optimal use of existing devices which will lead to improved clinical outcomes and more effective patient management and safety is a key feature. The manuscript management system is completely online and includes a quick and fair peer-review system. Visit http://www. dovepress.com/testimonials.php to read real quotes from authors. 